University of Vermont

UVM ScholarWorks

6-28-2014

\title{
Computational studies of multiple-particle nonlinear dynamics in a spatio-temporally periodic potential
}

\author{
Owen D. Myers \\ University of Vermont \\ Junru Wu \\ University of Vermont \\ Jeffrey S. Marshall \\ University of Vermont \\ Christopher M. Danforth \\ University of Vermont
}

Follow this and additional works at: https://scholarworks.uvm.edu/cemsfac

Part of the Human Ecology Commons, and the Medicine and Health Commons

\section{Recommended Citation}

Myers OD, Wu J, Marshall JS, Danforth CM. Computational studies of multiple-particle nonlinear dynamics in a spatio-temporally periodic potential. Journal of Applied Physics. 2014 Jun 28;115(24):244908.

This Article is brought to you for free and open access by the College of Engineering and Mathematical Sciences at UVM ScholarWorks. It has been accepted for inclusion in College of Engineering and Mathematical Sciences Faculty Publications by an authorized administrator of UVM ScholarWorks. For more information, please contact scholarworks@uvm.edu. 


\section{Computational studies of multiple-particle nonlinear dynamics in a spatio-temporally periodic potential}

Cite as: J. Appl. Phys. 115, 244908 (2014); https://doi.org/10.1063/1.4885895

Submitted: 30 April 2014 . Accepted: 18 June 2014 . Published Online: 30 June 2014

Owen D. Myers, Junru Wu, Jeffrey S. Marshall, and Christopher M. Danforth

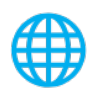

View Online

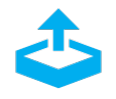

Export Citation

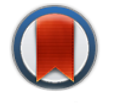

CrossMark

\section{ARTICLES YOU MAY BE INTERESTED IN}

Nonlinear dynamics of particles excited by an electric curtain

Journal of Applied Physics 114, 154907 (2013); https://doi.org/10.1063/1.4826267

Long-range interacting pendula: A simple model for understanding complex dynamics of charged particles in an electronic curtain device

Journal of Applied Physics 121, 154501 (2017); https://doi.org/10.1063/1.4980095

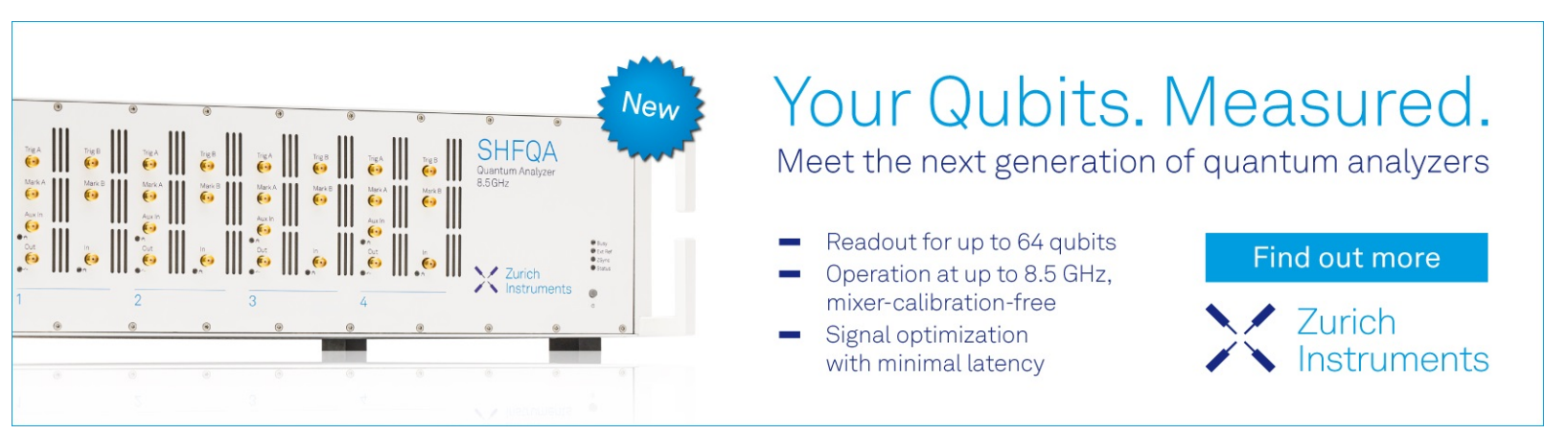




\title{
Computational studies of multiple-particle nonlinear dynamics in a spatio-temporally periodic potential
}

\author{
Owen D. Myers, ${ }^{1,2}$ Junru Wu, ${ }^{2,3,1, a)}$ Jeffrey S. Marshall, ${ }^{4,1}$ and Christopher M. Danforth ${ }^{5,6,1}$ \\ ${ }^{1}$ University of Vermont, Burlington, Vermont 05405, USA \\ ${ }^{2}$ Materials Science Program, University of Vermont, Burlington, Vermont 05405, USA \\ ${ }^{3}$ Department of Physics, University of Vermont, Burlington, Vermont 05405, USA \\ ${ }^{4}$ School of Engineering, University of Vermont, Burlington, Vermont 05405, USA \\ ${ }^{5}$ Department of Mathematics and Statistics, University of Vermont, Burlington, Vermont 05405, USA \\ ${ }^{6}$ Vermont Complex Systems Center, University of Vermont, Burlington, Vermont 05405, USA
}

(Received 30 April 2014; accepted 18 June 2014; published online 30 June 2014)

The spatio-temporally periodic (STP) potential is interesting in Physics due to the intimate coupling between its time and spatial components. In this paper, we begin with a brief discussion of the dynamical behaviors of a single particle in a STP potential and then examine the dynamics of multiple particles interacting in a STP potential via the electric Coulomb potential. For the multiple particles' case, we focus on the occurrence of bifurcations when the amplitude of the STP potential varies. It is found that the particle concentration of the system plays an important role; the type of bifurcations that occur and the number of attractors present in the Poincaré sections depend on whether the number of particles in the simulation is even or odd. In addition to the nonlinear dynamical approach, we also discuss dependence of the squared fractional deviation of particles' kinetic energy of the multiple particle system on the amplitude of the STP potential which can be used to elucidate certain transitions of states; this approach is simple and useful particularly for experimental studies of complicated interacting systems. (C 2014 AIP Publishing LLC.

[http://dx.doi.org/10.1063/1.4885895]

\section{INTRODUCTION}

Studies of nonlinear periodically driven systems are important to understanding the fundamental physics of many useful and interesting phenomena in applications of lasers, ${ }^{1}$ driven ratchets, ${ }^{2}$ hydrophilic particles on the surface of water waves ${ }^{3-5}$ Josephson junctions, ${ }^{6}$ etc. If bodies moving in periodically driven systems are allowed to interact, they can display a wealth of interesting physical phenomena associated with complex systems. ${ }^{7-9}$ Studies of the interaction among oscillators, bodies, nodes, etc., in periodic systems, and of how bodies in such systems collectively react to environmental forcing, can be valuable in a variety of fields from neuroscience ${ }^{10}$ to driven Josephson junction arrays. ${ }^{11}$

Interest in spatio-temporally periodic (STP) potentials began in 1951, when Kapitza published two papers ${ }^{12,13}$ on a planar pendulum with an oscillating suspension point, which is often referred to as the parametrically driven pendulum, or simply Kapitza's pendulum. The most interesting feature of this simple system is that under certain conditions, the pendulum stands stably in the inverted position. The change in stability of the inverted position through the oscillation of the suspension point is an example of what is known as dynamic stabilization, in which an inherently unstable system can be stabilized by periodic forcing.

The motion of a single particle immersed in a STP potential may be solved analytically using a simple approximation. For instance, for small $x$ we may approximate $f(\vec{x})$ with a harmonic oscillator potential resulting in equations of

${ }^{\text {a)} E l e c t r o n i c ~ m a i l: ~ J u n r u w u @ g m a i l . c o m ~}$ motion that can then be solved using Floquet theory. ${ }^{14}$ Linearization of the equation of motion in the limit of zero dissipation for one-dimensional motion allows the equation of motion to be expressed as the Hill equation, $\ddot{x}+g(t) x$ $=0$. If $g(t)=\cos (\omega t)$, this equation reduces to the Mathieu equation. ${ }^{15}$ Both the Hill and Mathieu equations have been studied extensively due to the interesting properties that they display and due to the many applications that can be associated with these equations, including the quantum pendulum, ${ }^{16}$ ion traps, ${ }^{17}$ and oscillations of a floating mass in a liquid. ${ }^{18}$ When the oscillations of $g(t)$ are fast compared to the natural frequency of oscillations when $g(t)$ is set to its maximum value, the method of averaging can be used. ${ }^{19,20}$

The interesting physical behavior of Kapita's stable inverted pendulum has enticed several researchers to study the nonlinear case both experimentally ${ }^{21-23}$ and numerically. ${ }^{22,24-29}$ For systems composed of particles in a STP potential, only a small number of publications have examined the nonlinear multiple particle dynamics accounting for the multi-particle interactions. Some examples of papers treating this subject include a study of the motion of hydrophobic/hydrophilic particles on the surface of Faraday waves, ${ }^{4,5}$ multiple charged particles in an STP potential generated by an electric curtain, ${ }^{30-32}$ and multiple particles in a periodically forced straining flow. ${ }^{33}$ These previous studies have considered very large numbers of particles and they have focused on the overall particle motion. In the current paper, we instead examine the dynamics of a relatively small number of particles in a STP potential using a dynamical systems point of view. Specifically, we seek to relate the 
nonlinear systems dynamics with multiple particles to the bifurcations and stability of single particles.

\section{METHODS}

The current computational study examines a onedimensional (1D) system with multiple particles interacting through a repulsive electrostatic $1 / r$ potential in an external STP potential field. The STP potential is

$$
\Phi=-A \cos x \cos t
$$

which produces equations of motion analogous to the parametrically driven pendulum in the horizontal plane. The coefficient $A$ is the potential amplitude, and the distance coordinate $x$ and time coordinate $t$ are non-dimensionalized using the wavenumber $k$ and the STP driving frequency $\omega$, respectively.

The driving force $\left(F_{\Phi}=-\nabla \Phi\right)$ has the form of a standing wave with oscillation amplitude $A$. The dimensionless wavelength $\lambda$ and the oscillation period $T$ are both equal to $2 \pi$. The system is assumed to be periodic over $n \lambda$, where $n$ is an integer, so we can define the concentration $\sigma$ as $N / n$ where $N$ is the number of particles in the simulation. For simplicity, all particles are assumed to carry the same charge and mass, where the non-dimensionalization is performed such that the dimensionless mass is equal to unity. Damping is proportional to particle velocity with a dimensionless damping coefficient $\beta$. In the current paper, we focus on the effect of the parameter $A$, and therefore maintain constant values of the other dimensionless parameters - the damping parameter and the dimensionless particle charge. These latter two parameters are set equal to $\beta=0.6$ and $q=1$ throughout the paper. These values for $\beta$ and $q$ are chosen because they are realistic for systems similar to those discussed in Refs. 30-32, after being dimensionalized.

The force on a particle located at $x_{i}$ imposed by a particle located at $x_{j}$, denoted by $F_{i j}$, is calculated with periodic boundary conditions. To address the forces imposed by long range interactions, we consider an infinite sequence of image systems using Ewald summation method, ${ }^{34}$ giving

$$
F_{i j}=\frac{q^{2} r_{i j}}{\left\|r_{i j}\right\|^{3}}+q^{2} \sum_{\nu=0}^{\infty} \frac{1}{\left(2 \pi \nu-r_{i j}\right)^{2}}-\frac{1}{\left(2 \pi \nu+r_{i j}\right)^{2}},
$$

where $r_{i j}=x_{i}-x_{j}$. The sum in (2) is convergent and may be written as a polygamma function $\psi^{m}(z)$ with series expansion

$$
\psi^{(m)}(z)=(-1)^{m+1} m ! \sum_{\nu=0}^{\infty} \frac{1}{(z+\nu)^{m+1}} .
$$

Using (3), we can express $F_{i j}$ as

$$
\begin{aligned}
F_{i j}= & \frac{q^{2} r_{i j}}{\left\|r_{i j}\right\|^{3}}-\left(\frac{q^{2}}{n \lambda}\right)^{2} \\
& \times\left(\psi^{(1)}\left(1+r_{i j} / \lambda\right)-\psi^{(1)}\left(1-r_{i j} / \lambda\right)\right) .
\end{aligned}
$$

The equation of motion for the $i$ th particle is given by

$$
\ddot{x}_{i}=-\beta \dot{x}_{i}+F_{\Phi}+\sum_{j \neq i}^{N} F_{i j}
$$

where the second term on the RHS is due to the imposed STP potential field and the third term on the RHS is the particle interactions. An example of the system containing seven particles may be found in Fig. 1.

\section{A. The phase space}

For $N$ particles in an autonomous system, the degrees of freedom (or dimension) of the phase space is $2 d N$, where $d$ is the dimension of the physical system. In STP problems, there is an explicit time dependence in the potential and therefore the system is non-autonomous. Non-autonomous systems may be transformed into autonomous form by introducing an extra degree of freedom, which in a first-order system is given by $x_{3}=t$. Though this seems to be a trivial representation of time, this autonomous formulation is necessary when distinguishing types of bifurcations. This augmented system thus has $2 d N+1$ degrees of freedom, which constitutes the "full phase space."

\section{B. Poincaré sections}

The standard choice for making Poincaré sections in driven systems is a time map taken at the system driving period. Time maps are stroboscopic views of a trajectory expressed as $x(t=2 \pi n)$ when $n$ is a positive integer. A Poincaré section includes any point where a continuous trajectory transversely intersects a subspace of the full phase space. ${ }^{35}$ Time maps, as defined above, will produce Poincaré sections with dimension one less than the dimension of the full phase space. In a time map, the path of a particle is always transverse to the $x-\dot{x}$ plane, and therefore a point of intersection of the trajectory with this plane is a convenient sub-space that satisfies the criterion necessary to be a Poincaré section.

\section{Kinetic energy fluctuations}

It is well known that as a system approaches a bifurcation point, it may take longer for transients of the relevant

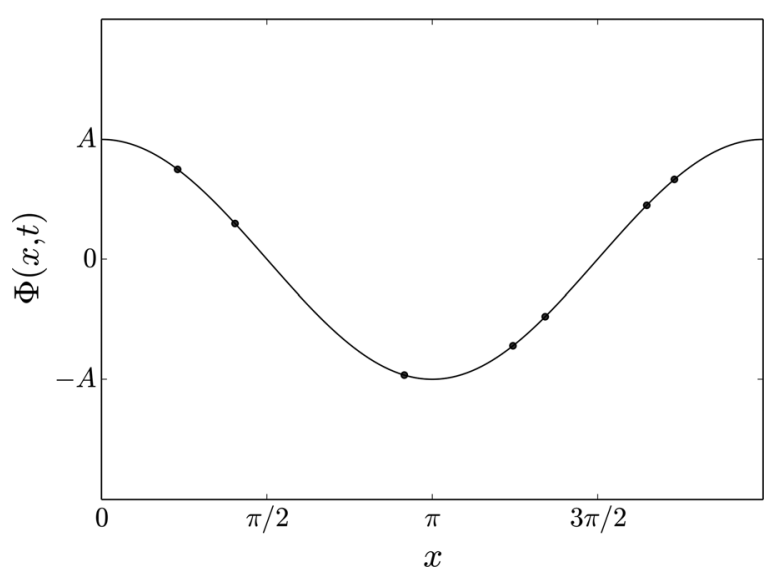

FIG. 1. Example of the system with $N=7$ and $A=2.758$, depicted as particles on the surface of a standing wave. (Multimedia view) [URL: http:// dx.doi.org/10.1063/1.4885895.1] 
quantity to die out or for the system to recover from an external perturbation. ${ }^{36}$ This behavior is known as the critical slowing down phenomena. Most real systems are subject to some natural perturbations, and these perturbations can become particularly apparent near the bifurcation points. Measuring the increase of the variance in a physical quantity can therefore be used as a method to predict the presence of a bifurcation point. ${ }^{36}$ The model system considered in the current paper has no external perturbations, aside from computer round-off error. In the limit $t \rightarrow \infty$, the damped system would be expected to settle into an attractor, but the finite time of real simulations ensures the presence of small fluctuations in "residual" transients. In other words, multiple particle systems have a "large" number of degrees of freedom, therefore some small trace of the initial transient behavior (residual transients) will most likely be detectable. The amount of residual transients may be found in the kinetic energy fluctuations. It is known that the kinetic energy fluctuations may contain some information about the "effective number of degrees of freedom." 37 The more degrees of freedom, the more residual transients will be present. This correspondence between the effective degrees of freedom and the kinetic energy fluctuations is what makes the kinetic energy fluctuations an interesting quantity to examine.

The square of the deviation of the particle kinetic energy is given by $(\Delta K E)^{2} \equiv\left\langle K E^{2}\right\rangle-\langle K E\rangle^{2}$,

$$
(\Delta K E)^{2}=\frac{1}{4} \sum_{i, j}^{N}\left(\left\langle v_{i}^{2} v_{j}^{2}\right\rangle-\left\langle v_{i}^{2}\right\rangle\left\langle v_{j}^{2}\right\rangle\right),
$$

and $v_{i}, v_{j}$ denote the $i$ th and $j$ th particle velocities, respectively. The average is calculated as $\langle K E\rangle^{2}=\frac{1}{4} \sum_{i, j}^{N}\left\langle v_{i}^{2}\right\rangle\left\langle v_{j}^{2}\right\rangle$. The normalized squared deviation of the kinetic energy is given by

$$
\delta_{K E} \equiv \frac{\Delta K E}{\langle K E\rangle^{2}}
$$

\section{RESULTS}

\section{A. Single particle overview}

The dynamics of a single particle immersed in the onedimensional STP potential $\Phi$, given by (1), are similar to the dynamics of the parametric pendulum. In this paper, we only discuss dynamics for the first bifurcation sequence leading to the chaotic regime, even though there are many consecutive regimes of stable limit cycles bifurcating into chaotic trajectories. In Fig. 2, the first bifurcation sequence is shown for an ensemble of initial conditions. Table I lists the type of bifurcations, the critical values of $A$ at which each bifurcation occurs, and the period of the limit cycle following each bifurcation. The table is truncated after the 6th bifurcation due to numerical resolution limitations for distinguishing bifurcation onset in a small volume of the phase space. For $0<A<A_{c 1}$, a particle will move toward and equilibrate at the antinodes of the potential $\Phi(x, t)$ (i.e., the maxima of $\cos x)$. As $A$ is increased, the fixed points in the Poincaré section bifurcate in a supercritical flip bifurcation leading to a

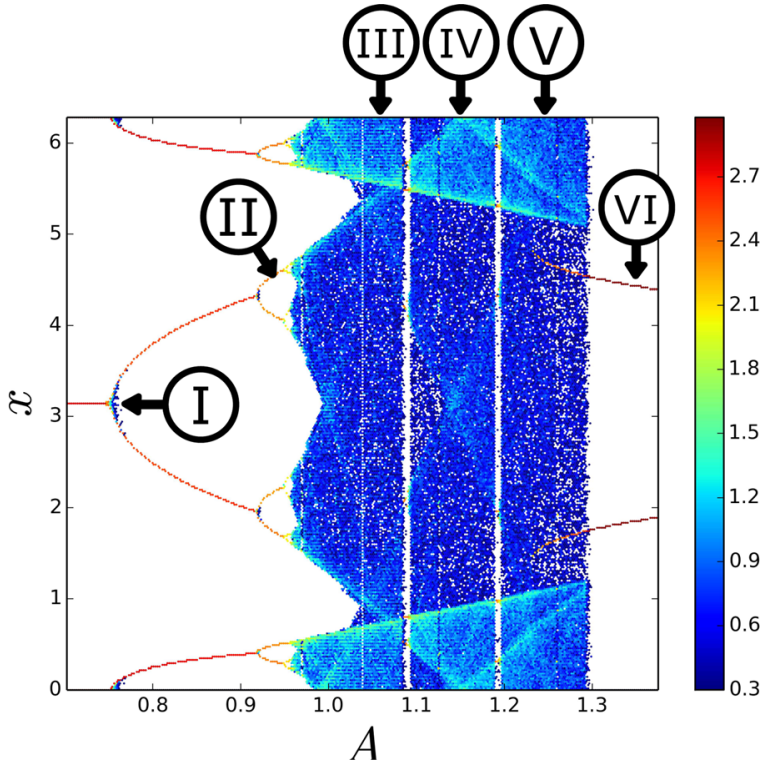

FIG. 2. Bifurcation diagram formed by taking a two-dimensional histogram $(300 \times 300$ bins $)$ of the final Poincaré section of 1830 trajectories with different initial conditions for 300 different values of $A$. The gray scale represents the base 10 logarithm of the number of particles in a bin. The Roman numerals are listed here for comparison with $\delta_{K E}$ shown in Fig. 3.

period-2 limit cycle for $A_{c 2}<A<A_{c 3}$. This transition is not a Hopf bifurcation because the explicit time dependence in the equations of motion must be considered as a degree of freedom to the phase space. Consequently, what might appear as a fixed point in the $x-\dot{x}$ phase space in a bifurcation diagram is actually a period- 1 trajectory in the full phase space. We prove this using Floquet theory, by numerically calculating the stability multipliers. Both of the two non-trivial stability multipliers have no imaginary component close to the bifurcation point. At the bifurcation, one stability multiplier becomes smaller than -1 while the other remains close to zero, indicating a period-doubling supercritical flip bifurcation. This stability multiplier passing through -1 is shown in Fig. 3(a), where it is denoted with a Roman numeral I. The values of $A$ for which the first six bifurcations occur, shown in Table I, indicate a period-doubling cascade route to chaos. The computed values yield a Feigenbaum constant of 4.00 with an upper error bound of 6.00 and a lower bound of 2.89. The accepted value of 4.669 for period-doubling bifurcations $^{38}$ is within the error bounds. The Feigenbaum constant $\mathcal{F}$ is evaluated with

$$
\mathcal{F}=\lim _{n \rightarrow \infty} \frac{A_{c n-1}-A_{c n-2}}{A_{c n}-A_{c n-1}},
$$

TABLE I. Bifurcations.

\begin{tabular}{lccc}
\hline \hline$A_{c n}$ & Bifurcation & $A \pm 5 \times 10^{-5}$ & New period \\
\hline$A_{c 1}$ & Supercritical flip & 0.75365 & 2 \\
$A_{c 2}$ & Cyclic fold & 0.91875 & 2 \\
$A_{c 3}$ & Supercritical flip & 0.94985 & 4 \\
$A_{c 4}$ & Supercritical flip & 0.95650 & 8 \\
$A_{c 5}$ & Supercritical flip & 0.95790 & 16 \\
$A_{c 6}$ & Supercritical flip & 0.95825 & 32 \\
\hline \hline
\end{tabular}



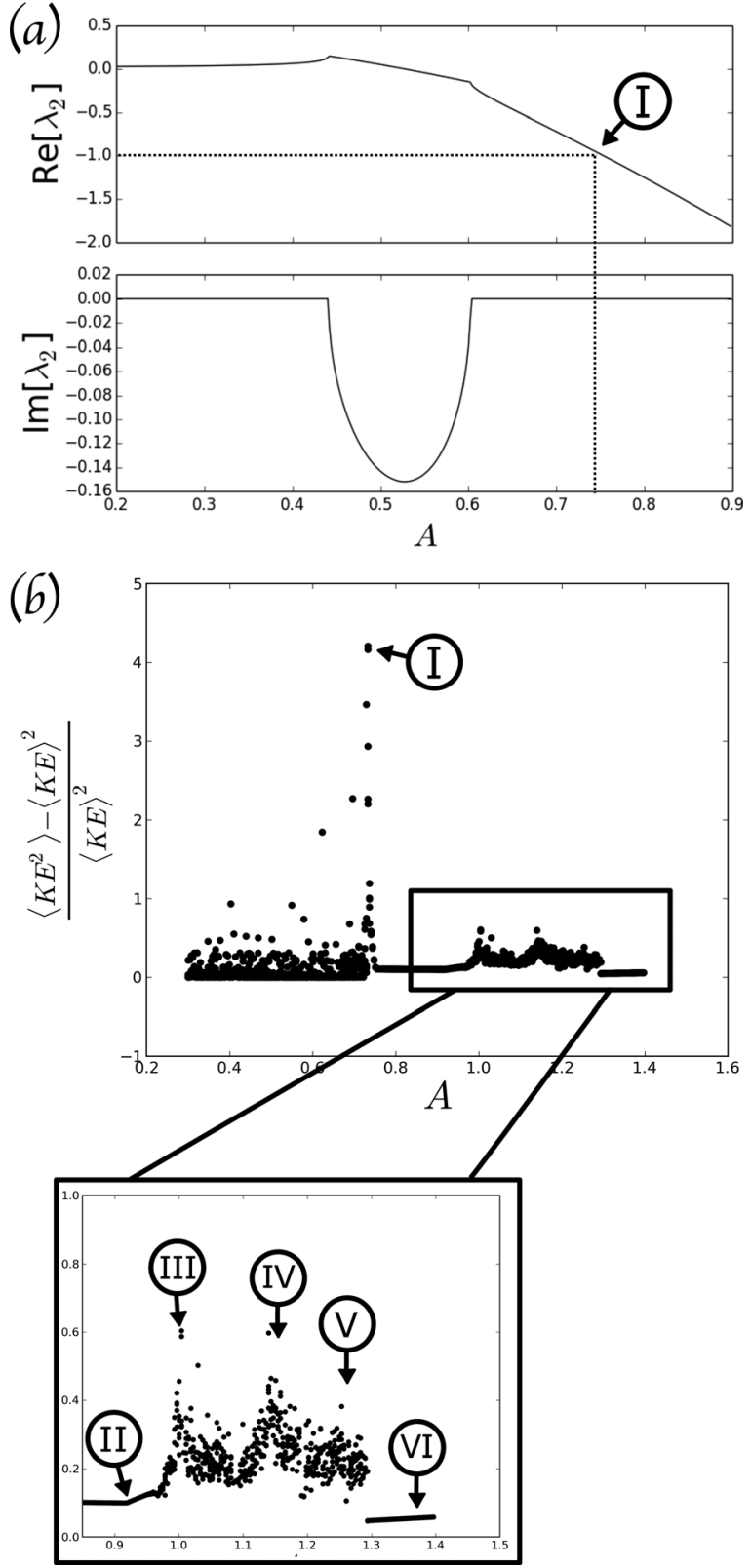

FIG. 3. Single particle: (a) One of the two Floquet stability multipliers for the $x=\pi$ fixed point as a function of the potential oscillation amplitude as it is increased through the first bifurcation point. (b) Kinetic energy fluctuations. Roman numerals for comparison with the bifurcation diagram in Fig. 2.

where $A_{c n}$ is the $n$th critical value of $A$ for which a perioddoubling bifurcation occurs. The two lines coming out of the chaotic region in Fig. 2 are each attractors representing stable propagating trajectories, one with a positive velocity and one with a negative velocity. These propagating trajectories travel across $\lambda$ once per period of the driving potential field.

\section{B. Kinetic energy fluctuations of one particle}

Before going to the multi-particle case, it is informative to compare the bifurcation diagram (Fig. 2) to the calculation of $\delta_{K E}$ for a single particle, which is shown in Fig. 3. We also show a Floquet stability analysis of the fixed point at $x=\pi$ through $A_{c 1}$ for comparison. Floquet stability analysis is a powerful tool in analyzing bifurcations, but it is not easily applied to multiple particle systems. It has been applied to coupled Kapitza pendulums by Ref. 39. For a description of single particle stability analyses, we refer the reader to Refs. 26 and 40, which are both studies of similar systems and use the Floquet technique to study bifurcations. In Fig. 3(a), the real and imaginary components of the stability multiplier that causes the bifurcation (one of the two complex Floquet stability multipliers $\lambda_{1}$ and $\lambda_{2}$ ) are plotted as $A$ is increased through $A_{c 1}$. In Fig. 3(b), $\delta_{K E}$ is plotted as $A$ is increased through the full range shown in the bifurcation diagram in Fig. 2.

In Figs. 2 and 3, the key regions associated with different system behaviors have been identified using Roman numerals. For small values of $A$, Fig. 3(b) shows a wide range of scattered points. However, the particle exhibits very little motion within this range of small $A$ values. As $A$ is increased, there exists a peak in the fluctuations near $A_{c 1}$, which is a consequence of the critical slowing down phenomenon (region I). As $A$ is increased past $A_{c 1}$, the fluctuation amplitude is relatively constant until $A$ approaches $A_{c 2}$, where a kink is observed (region II). When $A$ is in the chaotic and near-chaotic regimes (regions III, IV, V), the fluctuations increase in amplitude and are irregular, as shown in the inset in the figure. The two regions where the fluctuation amplitude decreases markedly in this inset correspond to the two periodic windows seen in Fig. 2. At the end of the chaotic regime, there is a discontinuous jump in the fluctuations to a comparatively small and relatively constant value (region VI). This last section shows the transition to propagating trajectories, and we will see that this feature is present in all cases where this transition occurs. Under closer inspection, region VI overlaps with region $\mathrm{V}$ because, just as in the bifurcation diagram, the propagating trajectories exist simultaneously with the chaotic regime for a small range of $A$.

\section{Integer concentrations}

The bifurcation diagrams for multiple interacting particles, with $N=2,3,4,5,6$, and 7, are shown in Fig. 4. The increased degrees of freedom that occur for $N>1$ make it difficult to investigate an ensemble of initial conditions that exhaustively fill the phase space. We use random positions distributed with even probability across $x$ (with $\dot{x}(0)=0$ ) as initial conditions for each run to explore a set of possible initial conditions. The bifurcation plots are made by taking the last Poincaré section after 150 driving cycles of a simulation, projecting it onto the position axis, and then plotting the positions against the value of $A$ used in that simulation. For very small $A$, the final Poincaré sections are scattered because, for these values, transients die out very slowly. For larger $A$, there are clearly defined points in the Poincaré sections that denote limit cycles in the full phase space. For the rest of the paper, the stable limit cycles in the Poincare sections are referred to more generally as attractors. At first glance, Fig. 4 appears to indicate a larger number of particles for odd values of $N$ than it does for even values of $N$. As $A$ is further increased, a bifurcation occurs for all the cases shown, although it is difficult to see in Fig. 4(e). For other 
(a)

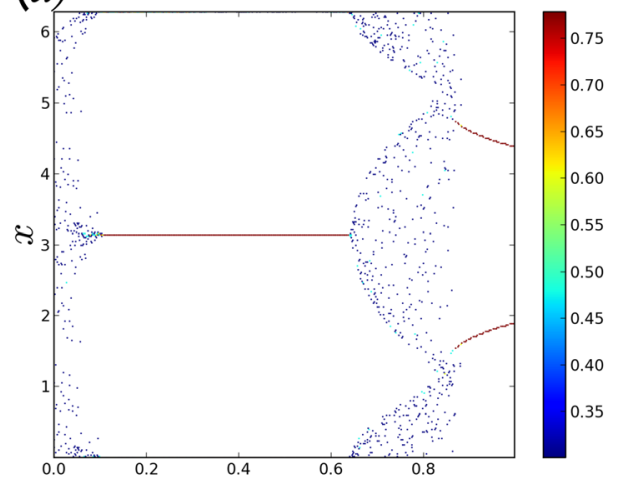

(d)
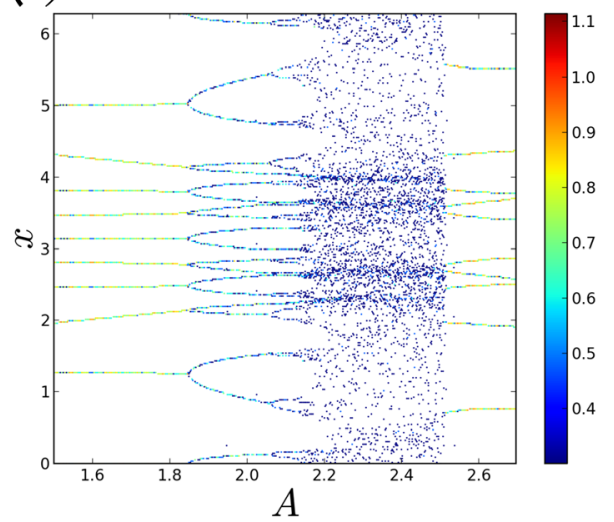

(b)

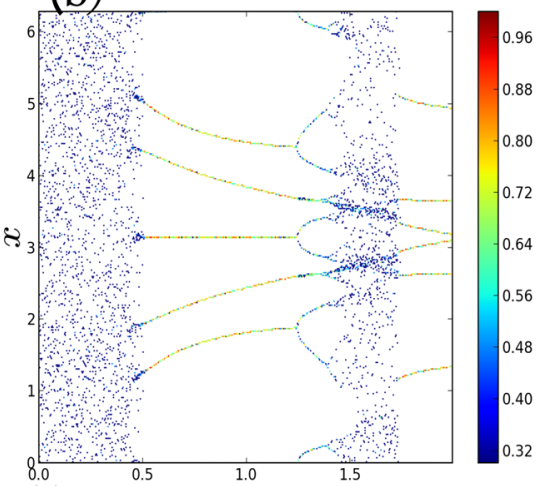

(e)

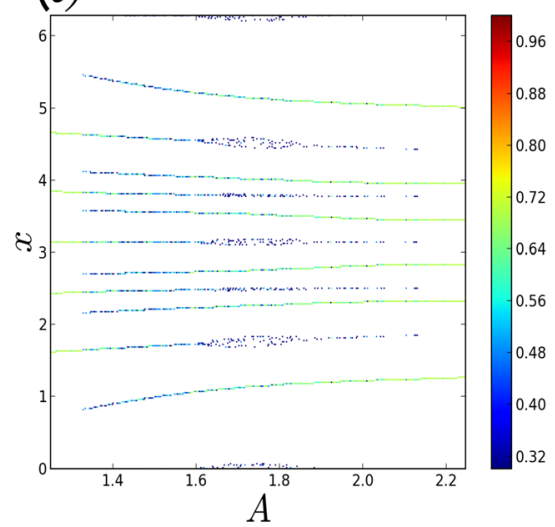

(c)

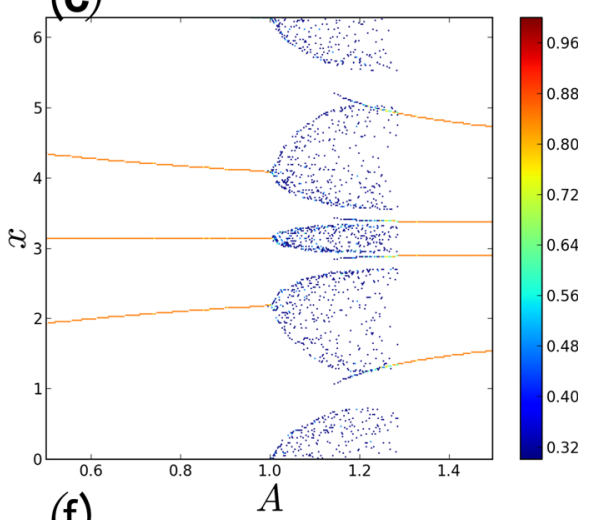

(f)

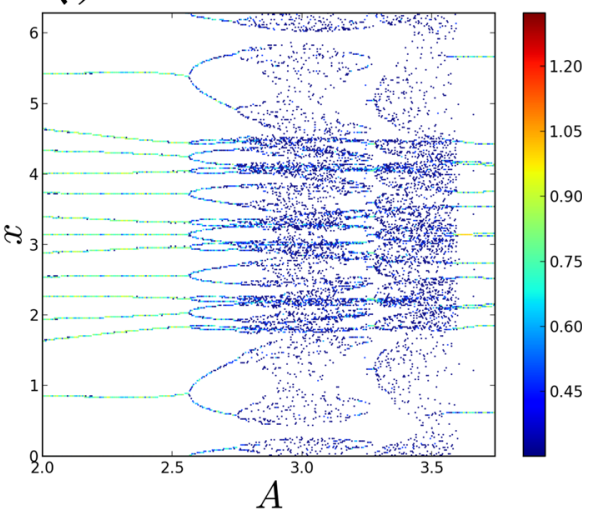

FIG. 4. Multiple particle bifurcation diagrams made by: (1) initializing particles at random initial positions with $\dot{x}(0)=0$, (2) projecting the Poincaré section onto the position axis after 150 cycles and plotting against the value of $A$, and (3) repeating the process for 400 values of $A$ in the range of interest. The number of particles used in each bifurcation diagram is (a) $N=2$, (b) $N=3$, (c) $N=4$, (d) $N=5$, (e) $N=6$, (f) $N=7$. Note that the range of A values explored differs in each figure.

values of $A$, the diagrams in Fig. 4 appear as scattered points, implying either chaotic motion, high-period trajectories, or motion on a torus (after a Neimark bifurcation). Past this scattered regime, the system collapses into a new stable regime that is qualitatively similar to the propagating trajectories that occur after the chaotic regime in the single particle case.

The discrepancy between the number of possible attractors for odd and even values of $N$ can be explained by considering the relationship between the number of particles and the number of antinodes in one period of the potential. For the trajectory $\left(x_{1}(t), \ldots, x_{2 d N}(t)\right)$, the number of attractors that are observed for values of $A$ before the first bifurcation is equal to the number of particles when $N$ is even and twice the number of particles when $N$ is odd. In other words, there is one possible final state configuration of the whole system when $N$ is even and two possible final state configurations of the system when $N$ is odd. To see why this occurs, we examine in detail the cases of three and four particles per period. Figs. 5(a)-5(d) show cartoons of the final particle configurations for cases $N=4$ and $N=3$, respectively. In these cartoons, the particle position in the periodic domain is drawn as an angle, so that motion of the particle in the $x$ domain corresponds in the figure to rotation about a circle. The intersection points of the circle with a horizontal bisection line occur at the antinodes of the potential $\Phi$. In this figure, at least one particle can always be found at the antinode of $\Phi$. From the single particle case, we know the antinodes can act as attractors, where individual particles remain motionless in the $x-\dot{x}$ phase plane. In the case of four particles, shown in Fig. 5(a), two particles may occupy the antinodes of $\Phi$ and the other two particles oscillate about the (a)

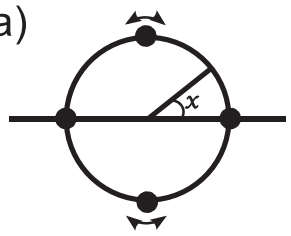

(c)

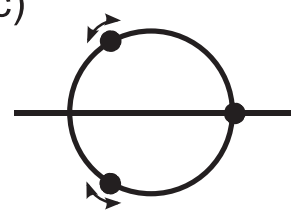

(b)

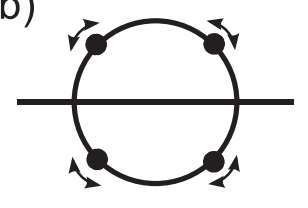

(d)

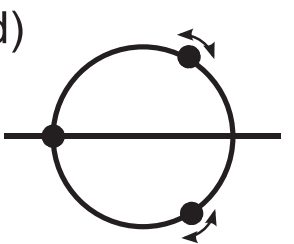

FIG. 5. The position along the periodic domain is indicated by an angle around a circle. The black dots represent average particle positions before the first bifurcation. The line bisecting the circle passes through the two antinodes of the potential field. Part (a) shows the $N=4$ stable configuration which is an asymptotically stable fixed point in the Poincaré sections of the full phase space. Part (b) is an $N=4$ unstable configuration which is the unstable fixed point in the Poincaré sections of the full phase space. Parts (c) and (d) show two different possible stable configurations for $N=3$, both of which are stable fixed points in the Poincaré sections of the full phase space. 
nodes of $\Phi$. One might imagine that a rotation of this configuration by $\pi / 4$ (shown in Fig. 5(b)) might be a fixed point in the Poincare section of the full phase space, and indeed it is, but it is not a stable configuration and, unless perfectly configured, it will collapse into the configuration shown in Fig. 5(a). For three particles, one particle sits at antinode and the two remaining particles compete over the other antinode, as shown in Figs. 5(c) and 5(d). Which antinode a particle is attracted to depends on the initial conditions. In both the four and three particle cases, the particles at the antinodes are stationary.

Drawing lines between the adjacent average particle positions in the circular topology creates a regular convex polygon inscribing the circle. From our description of the particle behavior above, at least one vertex of the polygon must be at an antinode. For a regular polygon inscribing the circle with an even number of vertices, each antinode may touch a vertex and it is symmetric under all rotations obeying this rule. For a polygon with an odd number of vertices, however, only one vertex can occupy an antinode so that rotations by $\pi / N$ flip the symmetry about a line vertically bisecting the circle. There are always two unique possible stable configurations when $N$ is odd, but only one when $N$ is even. This picture changes for even values of $N$ when $N>6$. For $N>6$, the stable configuration no longer occurs for a pair of particles located at each antinode, but rather for pairs oscillating on either side of the antinodes much like what is shown in Fig. 5(b) but with an extra particle on the top and bottom of the circle.

In Fig. 6, the value of $A$ at which the first bifurcation occurs is plotted as a function of $N$, with separate lines for $N$ even (red squares) and $N$ odd (blue triangles). A striking characteristic of Fig. 6 is that between $N=6$ and $N=8$, there is a cross-over point at which the even $N$ line jumps upward

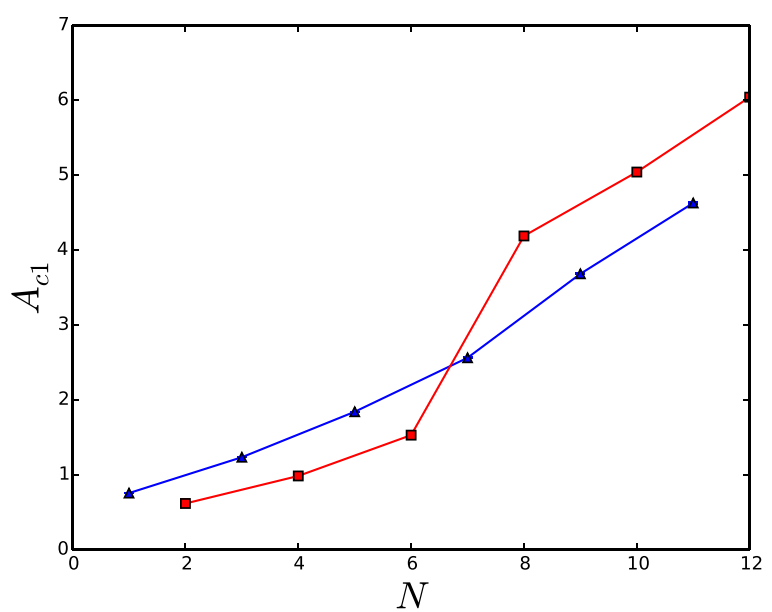

FIG. 6. A plot of the value of $A$ at which the system first bifurcates $\left(A_{c 1}\right)$ as a function of the number of particles in simulation $(N)$. Even particle numbers are shown with red squares and odd particle numbers are shown with blue triangles. A cross-over between the $N$ odd and $N$ even curves occurs between $N=6$ and $N=7$, which corresponds to the first bifurcation for the even value of $N$ changing from a Neimark bifurcation to a cyclic fold bifurcation. The $N$ odd bifurcations are all supercritical flip bifurcations for the values of $N$ shown here. The upper (lower) error bound is the value of $A$ which we are certain is after (before) the bifurcation. The error bounds are seen to be quite small in the figure. and crosses through the odd $N$ line. This sudden jump in the even $N$ line between $N=6$ and $N=8$ occurs due to a change in type of first bifurcation. The first bifurcation when $N=6$ as well as the first bifurcations for all lower even values of $N$ are Neimark bifurcations (a.k.a bifurcation to a torus) in which $N$ stability multipliers cross the unit circle with nonzero imaginary components. Half of those stability multipliers $(N / 2)$ that cross the unit circle have positive imaginary components and the other half have the complementary negative imaginary components. For $N=8$ and all higher even values of $N$, the first bifurcation becomes a cyclic fold bifurcation, although the bifurcations for odd values of $N$ remain supercritical flip bifurcations.

We can qualitatively understand the transition which occurs when $N$ is changed from six to eight in Fig. 6 by observing how the bifurcation diagrams, and therefore the stable attractors, depend on the particle number. In Fig. 4(e), after the first bifurcation, the particle motions are quenched by the presence of other attractors. For the sake of discussion, we distinguish the two competing sets of attractors based on whether they are found at the far left or far right edges of the diagrams, respectively. Comparing the cases of $N=2, N=4$, and $N=6$ in Fig. 4, we see similar rightmost attractors that appear abruptly at different values of $A$ in each case. As $N$ (even) increases, the rightmost attractors increasingly impinge on the leftmost attractors. This impingement is responsible for the crossing of the lines in Fig. 6. When $N$ increases from six to eight, the rightmost attractors extinguish the left most attractors and become the first available attractors for $N \geq 8$ (even). These new first attractors, previously the rightmost, represent a fundamentally different type of limit cycle in the full phase space than what was previously first available. Therefore, when this attractor first bifurcates, it falls outside of the original progression, causing the jump in $A_{c 1}$ as $N$ is changed from six to eight shown in Fig. 6.

For all of the cases shown in Fig. 4, the system eventually collapses back into clearly defined attractors which have the form of "propagating" trajectories, as was also observed for the single particle case. These attractors display non-zero net particle motion of one particle when $N$ is odd, but no net motion when $N$ is even. The particles travel in either the $\pm x$ direction before a collision-like event. After the "collision," they travel in the opposite direction having exchanged some kinetic energy with the particle with which the collision occurred. When $N$ is odd the transport of one particle occurs either in the $\pm x$ direction depending on the initial conditions. There is no possible counter-propagating particle pair for one of the particles with $N$ odd.

In Fig. 7, the squared fractional deviation of the kinetic energy $\delta_{K E}$ is plotted for all of the bifurcation diagrams shown in Fig. 4. These plots all exhibit a discontinuity at the point corresponding to transition to a state with the propagating trajectories. The discontinuity is not as clear in Fig. 7(e) because (as can be observed in the corresponding bifurcation diagram Fig. 4(e)) the propagating trajectory begins before the first bifurcation. In Fig. 7(e), the curve starting below and crossing at $A \approx 1.75$ indicates the values of $\delta_{K E}$ for the propagating trajectory. 

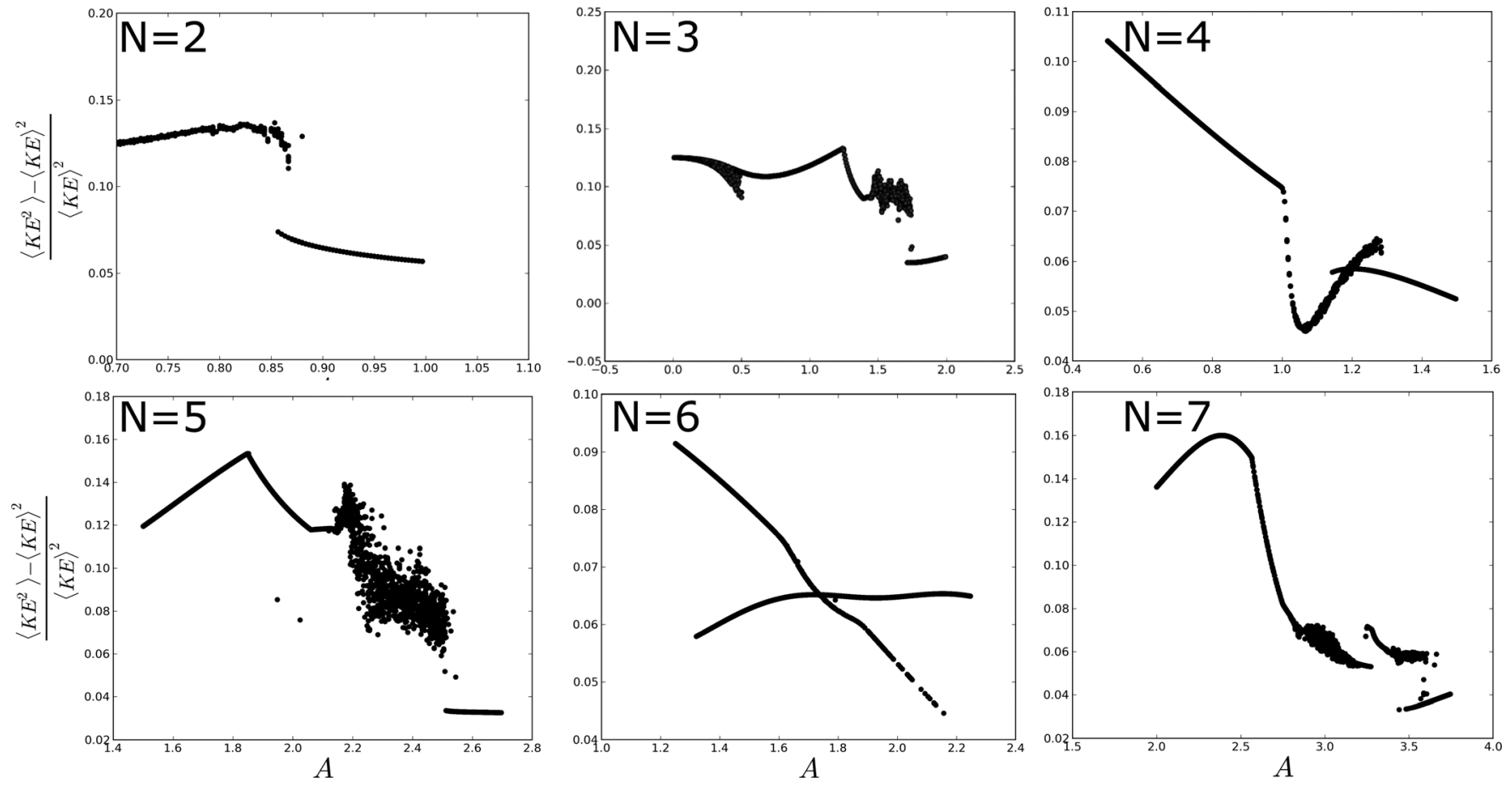

FIG. 7. Kinetic energy fluctuations for various particle counts.

\section{Scaling}

When the periodicity of the system is increased $(n>1)$, two distinctly different possibilities exist. One possibility is an integer concentration, over a larger periodic system (e.g., $n=2$ and $N=4: \sigma=2$ ). The second possibility is a fractional concentration (e.g., $n=2$ and $N=3: \sigma=1.5$ ). When considering long-range particle interactions, it is reasonable to assume that increasing the system size might change the dynamics even if the concentration is held fixed. The first possibility above results in system dynamics very similar to those discussed in the current paper, whereas the second possibility would result in a completely different symmetry of the system having very different dynamics. The effect of period number $n$ on the system dynamics was examined in the current study by running simulations of equal concentration over larger system sizes (i.e., large values of $n$ ). The system dynamics in these larger systems is observed to be qualitatively the same as for the smaller system sizes reported in the paper, although the exact values of $A$ for which bifurcations occur is observed to change slightly. We find that for concentrations larger than two, the effect of scaling the system size while maintaining the concentration is negligible even when comparing critical $A$ values.

\section{CONCLUSION}

We investigate the dynamics of multiple particles with long-range interactions in a STP system by examining Poincaré sections and fluctuations of the kinetic energy $\left(\delta_{K E}\right)$ for different numbers of particles. Our results are fundamentally interesting because of their importance in understanding complexity in time-dependent systems. The possible dynamics that exist in a wide range of different system configurations make the problem challenging, but even in the small area of the parameter space discussed in this paper we have found a variety of interesting dynamics. For instance, it is shown that the particle number $N$ influences the stability and the number of possible final states in a system having integer concentrations. The possible limit cycles of the system are shown to be sensitive to whether $N$ is even or odd, and the influence of the particle number on the type of bifurcation is discussed. The squared fractional deviation kinetic energy is examined as a function of the potential amplitude $\left(\delta_{K E}(A)\right)$, and it is found to exhibit interesting features at and near transition points. In particular, discontinuities in $\frac{d\left(\delta_{K E}\right)}{d A}$ and $\delta_{K E}$ mark transitions between oscillatory and propagating modes, respectively. The measure $\delta_{K E}$ may be useful for future experimental investigations of these systems.

Our work has demonstrated interesting and complex behaviors of multiple particles with the Coulomb interaction in a STP potential. In particular, the dynamics of the system is sensitive to particle concentration and the dynamics can be described by the squared fractional deviation of the particle kinetic energies. The latter is particularly valuable for studying bifurcations in real systems. For example, in the aforementioned studies of the motions of hydrophobic/ hydrophilic particles on the surface of Faraday waves, the particles will interact due to capillary forces caused by their distortion of the local surface of the water, rather than through the Coulomb interaction, which leads to particle clustering. ${ }^{3}$ It may be convenient to study this type of behavior using the squared fractional deviation of the systems kinetic energy because this measure will decrease in the event of clustering as it measures the effective number of degrees of freedom. ${ }^{37}$

Studies of multiple charged particles in a standing-wave electric curtain and in acoustic waves are also important areas of research for applications of dust-particle mitigation, 
e.g., from a solar panel. ${ }^{31}$ Charged particles interacting in standing-wave electric curtains and standing-wave acoustic fields exhibit complicated dynamics that may be illuminated by studying the squared fractional deviation of the particle kinetic energy. For example, in Ref. 41, it was observed that for charged particles in a standing-wave acoustic field, relative motion of smaller particles is faster than that of larger particles, so that the large particles act as collectors within some agglomeration volume. Any small particles present in the agglomeration volume are likely to aggregate to a larger particle, and this aggregation is desirable for applications such as cleaning particles from surfaces. A sweep of the acoustic driving parameters to find the configuration for which maximal aggregations occurs could clearly be found and described in terms of the minimal squared fractional deviation of the particle kinetic energies, again due to its measure of the effective number of degrees of freedom. In general, we hope our work may stimulate further research of STP systems with interacting particles and shed some light on their complicated and exciting dynamics.

\section{ACKNOWLEDGMENTS}

This work was supported by NASA Space Grant Consortium under Grant Nos. NNX10AK67H, NNX08AZ07A, and NNX13AD40A. We also want to acknowledge the Vermont Advanced Computing Core which was supported by NASA (NNX 06AC88G), at the University of Vermont for providing the high performance computing resources used for the work in this paper.

${ }^{1}$ T. Simpson, J. Liu, and A. Gavrielides, "Period-doubling cascades and chaos in a semiconductor laser with optical injection," Phys. Rev. A 51, 4181-4185 (1995).

${ }^{2}$ P. Olbrich, J. Karch, E. L. Ivchenko, J. Kamann, B. März, M. Fehrenbacher, D. Weiss, and S. D. Ganichev, "Classical ratchet effects in heterostructures with a lateral periodic potential," Phys. Rev. B 83, 165320 (2011).

${ }^{3}$ N. Francois, H. Xia, H. Punzmann, and M. Shats, "Inverse energy cascade and emergence of large coherent vortices in turbulence driven by faraday waves," Phys. Rev. Lett. 110, 194501 (2013).

${ }^{4}$ P. Denissenko, G. Falkovich, and S. Lukaschuk, "How waves affect the distribution of particles that float on a liquid surface," Phys. Rev. Lett. 97, 244501 (2006).

${ }^{5}$ G. Falkovich, A. Weinberg, P. Denissenko, and S. Lukaschuk, "Floater clustering in a standing wave," Nature 435, 1045 (2005).

${ }^{6}$ E. Boukobza, M. G. Moore, D. Cohen, and A. Vardi, "Nonlinear phase dynamics in a driven bosonic josephson junction," Phys. Rev. Lett. 104, 240402 (2010)

${ }^{7}$ P. Bak, C. Tang, and K. Wisenfeld, "Self-organized criticality: An explanation of 1/f noise," Phys. Rev. Lett. 59, 381 (1987).

${ }^{8}$ P. Bak, K. Chen, and M. Creutz, "Self-organized criticality in the game of life," Nature 342, 780 (1989).

${ }^{9}$ E. A. Martens, S. Thutupalli, A. Fourriere, and O. Hallatchek, "Chimera states in mechanical oscillator networks," Proc. Natl. Acad. Sci. U. S. A. 110, 10563-10567 (2013).

${ }^{10}$ M. I. Rabinovich, P. Varona, A. I. Selverston, and H. D. I. Abarbanel, "Dynamical principles in neuroscience," Rev. Mod. Phys. 78, 1213-1265 (2006).

${ }^{11}$ A. J. Rimberg, T. R. Ho, I. M. C. Kurdak, J. Clarke, K. L. Campman, and A. C. Gossard, "Dissipation-driven superconductor-insulator transition in a two-dimensional josephson-junction array," Phys. Rev. Lett. 78, 2632-2635 (1997).

${ }^{12}$ P. L. Kapitza, "Dynamical stability of a pendulum when its point of suspension vibrates," Zh. Eksp. Teor. Fiz. 21, 588 (1951).

${ }^{13}$ P. L. Kapitza, "Pendulum with a vibrating suspension," Usp. Fiz. Nauk 44, 7-20 (1951); available at http://ufn.ru/ru/articles/1951/5/c/.

${ }^{14}$ S. Lefschetx, Differential Equations Geometric Theory, 2nd ed. (Dover Publications, New York, 1977).

${ }^{15}$ N. W. McLachlan, Theory and Application of Mathieu Functions, 1 st ed. (Oxford University Press, London, 1947).

${ }^{16}$ T. Pradhan and A. V. Khare, "Plane pendulum in quantum mechanics," Am. J. Phys. 41, 59-66 (1973).

${ }^{17}$ D. Leibfried, R. Blatt, C. Monroe, and D. Wineland, "Quantum dynamics of single trapped ions," Rev. Mod. Phys. 75, 281-324 (2003).

${ }^{18}$ L. Ruby, "Applications of the Mathieu equation," Am. J. Phys. 64, 39-44 (1996).

${ }^{19}$ N. N. Bogoljubov and Y. A. Mitropolski, Asymptotic Methods in the Theory of Nonlinear Oscillations (Gordon and Breach, New York, 1961).

${ }^{20}$ L. Dálessio and A. Polkovnikov, "Many-body energy localization transition in periodically driven systems," Ann. Phys. 333, 19-33 (2013).

${ }^{21}$ J. Starrett and R. Tagg, "Control of a chaotic parametrically driven pendulum,” Phys. Rev. Lett. 74, 1974-1977 (1994).

${ }^{22}$ R. Chacon and L. Marcheggiani, "Controlling spatiotemporal chaos in chains of dissipative kapitza pendula," Phys. Rev. E 82, 016201 (2010).

${ }^{23}$ D. Maravall, C. Zhou, and J. Alonso, "Hybrid fuzzy control of the inverted pendulum via vertical forces," Int. J. Intell. Syst. 20, 195-211 (2005).

${ }^{24}$ J. A. Blackburn, "Stability and hopf bifurcations in an inverted pendulum," Am. J. Phys. 60, 903 (1992).

${ }^{25}$ W. Szemplinska-Stupnicka, E. Tyrkiel, and A. Zubrzycki, "The global bifurcations that lead to transient tumbling chaos in a parametrically driven pendulum," Int. J. Bifurcation Chaos 10(9), 2161-2175 (2000).

${ }^{26} \mathrm{~S}$. Kim and B. Hu, "Bifurcations and transitions to chaos in an inverted pendulum," Phys. Rev. E 58, 3028-3035 (1998).

${ }^{27}$ M. V. Bartuccelli, G. Gentile, and K. V. Georgiou, "On the dynamics of a vertically driven damped planar pendulum,” R. Soc. 457, 3007-3022 (2001).

${ }^{28}$ E. Butikov, "Subharmonic resonances of the parametrically driven pendulum," J. Phys. A: Math. Gen. 35, 6209 (2002).

${ }^{29}$ R. W. Leven and B. P. Koch, "Chaotic behaviour of a parametrically excited damped pendulum," Phys. Lett. 86A, 71 (1981).

${ }^{30}$ S. Masuda, K. Fujibayashi, K. Ishida, and H. Inaba, "Confinement and transportation of charged aerosol clouds via electric curtain," Electr. Eng. Jpn. 92, 43 (1972).

${ }^{31}$ G. Liu and J. Marshall, "Particle transport by standing waves on an electric curtain,” J. Electrost. 68, 289-298 (2010).

${ }^{32}$ J. Chesnutt and J. S. Marshall, "Simulation of particle separation on an inclined electric curtain,” IEEE Trans. Ind. Appl. 49, 1104-1112 (2013).

${ }^{33}$ J. S. Marshall, "Particle clustering in periodically forced straining flows," J. Fluid Mech. 624, 69 (2009).

${ }^{34} \mathrm{P}$. Gibbon and G. Sutmann, "Long-range interactions in many-particle simulation," Quantum Simulations of Complex Many-Body Systems: From Theory to Algorithms, edited by J. Grotendorst, D. Marx and A. Muramatsu, (John von Neumann Institute for Computing, Jülich, 2002), NIC Series Vol. 10, pp. 467-506.

${ }^{35}$ J. Guckenheimer, Nonlinear Oscillations, Dynamical Systems, and Bifurcations of Vector Fields (Springer-Verlag, New York, 1983).

${ }^{36}$ M. Scheffer, J. Bascompte, W. A. Brock, V. Brovkin, S. R. Carpenter, V. Dakos, H. Held, E. H. V. Nes, M. Rietkerk, and G. Sugihara, "Early-warning signals for critical transitions," Nature 461, 53-59 (2009).

${ }^{37}$ F. Cecconi, F. Diotallevi, U. M. B. Marconi, and A. Puglisi, "Fluid-like behavior of a one-dimensional granular gas," J. Chem. Phys. 120, 35-42 (2004).

${ }^{38}$ J. Thompson and H. Stewart, Nonlinear Dynamics and Chaos, 2nd ed. (Wiley, New York, 2002).

${ }^{39}$ S.-Y. Kim and B. Hu, "Critical behavior of period doublings in coupled inverted pendulums," Phys. Rev. E 58, 7231-7242 (1998).

${ }^{40}$ O. D. Myers, J. Wu, and J. S. Marshall, "Nonlinear dynamics of particles excited by an electric curtain,” J. Appl. Phys. 114, 154907 (2013).

${ }^{41} \mathrm{D}$. Chen and J. Wu, "Dislodgement and removal of dust-particles from a surface by a technique combining acoustic standing wave and airflow," J. Acoust. Soc. Am. 127, 45-50 (2010). 